\title{
Whole-Genome Sequence Analysis of CTX-M Containing Escherichia coli Isolates from Retail Meats and Cattle in the United States
}

\author{
Daniel A. Tadesse,, Cong Li, Sampa Mukherjee, Chih-Hao Hsu, Sonya Bodeis Jones, \\ Stuart A. Gaines, Claudine Kabera,, Guy H. Loneragan,2 Mary Torrence, Dayna M. Harhay, \\ Patrick F. McDermott, ${ }^{1}$ and Shaohua Zhao ${ }^{1}$
}

In recent years, there have been increased reports on the detection of extended-spectrum beta-lactamase (ESBL)producing Escherichia coli and Salmonella strains from food-producing animals and animal products in the United States. We characterized 18 ESBL $E$. coli isolates from cattle $(n=5)$, chicken breast $(n=5)$, ground turkey $(n=6)$, ground beef $(n=1)$, and pork chops $(n=1)$ that were collected by the National Antimicrobial Resistance Monitoring System (NARMS) between 2011 and 2015. In vitro antimicrobial susceptibility testing was done against a panel of 14 antimicrobials followed by a secondary panel of $9 \beta$-lactam agents. Whole-genome sequencing was used to characterize the resistome, plasmids, and the genetic structures of the ESBL genes. All ESBL-producing $E$. coli isolates were resistant to at least three antimicrobial classes and carried various $b a_{\mathrm{CTX}} \mathrm{M}$ genes. Most of the cattle and ground turkey isolates carried $b l a_{\mathrm{CTX}-\mathrm{M}-27}$. In chicken breast isolates, $b l a_{\mathrm{CTX}-\mathrm{M}-1}$ was present as part of an ISEcpl transposition unit carried on a plasmid that shares sequence similarity with the backbone structure of the IncI plasmid. Isolates carrying the $b l a_{\mathrm{CTX}-\mathrm{M}-14}$ and $b l a_{\mathrm{CTX}-\mathrm{M}-15}$ genes, widely distributed in human clinical isolates, were also isolated. To our knowledge, this is the first report of the widely distributed $b l a_{\mathrm{CTX}-\mathrm{M}-14}$ and $b l a_{\mathrm{CTX}-\mathrm{M}-15}$ in $E$. coli isolates from retail meat samples in the United States. Different insertional sequences were identified upstream of these $b l a_{\mathrm{CTX} \text {-Ms }}$, including ISEcp1, IS26, and IS903-D. CTX-M in E. coli from food animals and retail chicken breast were often present on plasmids with other resistance genes. Other resistance genes identified

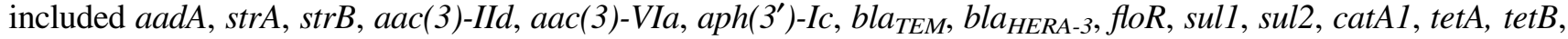
$d f r A$, and $q a c E$. These data describe the emergence of CTX-M-carrying E. coli isolates in food animals and animal products monitored by NARMS program.

Keywords: Escherichia coli, ESBL, CTX-M

\section{Introduction}

$\mathbf{E}$ XTEndED-SPECTRUm Beta-lactamases (ESBLs) are the most common cephalosporin resistance mechanism reported in members of the Enterobacteriaceae family. ${ }^{1}$ ESBLs are a group of enzymes with the ability to hydrolyze oxyimino-cephalosporins and thus cause resistance to cefotaxime, ceftazidime, ceftriaxone, cefuroxime, and cefepime, as well as monobactams (e.g., aztreonam). ${ }^{2}$ The introduction of extended-spectrum cephalosporins in clinical practice in the 1970 s was soon followed by reports of resistant strains of Enterobacteriaceae producing ESBLs. ${ }^{3}$ Since then, the occurrence of infection due to ESBL-resistant Enterobacteriaceae has rapidly increased and has become a major problem worldwide. In the United States, the Centers for Disease Control and Prevention estimates 26,000 infections and 1,700 deaths annually due to ESBL-producing Enterobacteriaceae. ${ }^{4}$

CTX-M-producing Escherichia coli have been associated with both hospital-acquired and community infections, mostly in urinary tract infections and bacteremia ${ }^{5-8}$ Studies over the last decade have shown that CTX-M-type ESBLs

\footnotetext{
${ }^{1}$ Division of Animal and Food Microbiology, U.S. FDA, CVM, Laurel, Maryland.

${ }^{2}$ Texas Tech University, Department of Animal and Food Science, Lubbock, Texas.

${ }^{3}$ U.S. FDA-CFSAN, Office of Applied Research and Safety Assessment (OARSA), Laurel, Maryland.

${ }^{4}$ USDA-ARS, U.S. Meat Animal Research Center, Meat Safety and Quality Research Unit, Clay Center, Nebraska.
}

(C) Daniel A. Tadesse, et al., 2018; Published by Mary Ann Liebert, Inc. This Open Access article is distributed under the terms of the Creative Commons License (http://creativecommons.org/licenses/by/4.0), which permits unrestricted use, distribution, and reproduction in any medium, provided the original work is properly cited. 
have become the predominant enzyme type in many parts of the world, ${ }^{9}$ and have spread rapidly through clinical populations of Enterobacteriaceae. ${ }^{10}$ Reports from several countries describe the presence of CTX-M-producing E. coli strains in apparently healthy food animals ${ }^{11-13}$ and food animal products, ${ }^{12}$ as well as pets ${ }^{14}$ and wild birds. ${ }^{15}$ Although CTX-Mproducing strains appear to have quickly spread worldwide, the increased prevalence of $E$. coli carrying these $\beta$ lactamases in numerous U.S. hospitals became apparent in the early 2000 s. $^{7,10,16}$

Intestinal carriage of CTX-M-producing bacteria in foodproducing animals and contamination of retail meat might contribute to increased occurrences of infections with ESBL-producing bacteria in humans. A study on the presence of indistinguishable E. coli genotypes carrying CTX-M genes obtained from poultry, poultry products, and human clinical samples in the Netherlands has suggested the possible exchange of these genes through the food chain. ${ }^{12}$ In the United States, few CTX-M ESBLs have been reported from food animals and animal products. ${ }^{11,17-19}$ The National Antimicrobial Resistance Monitoring System (NARMS) monitors changes in antimicrobial susceptibilities of zoonotic foodborne bacteria to medically important antimicrobials, including $\beta$-lactam antibiotics. Whole-genome sequencing has improved our ability to monitor resistomes and helps to identify and characterize emerging resistance genes and mobile genetic elements that facilitate the spread of these genes. The aim of this study was to investigate and characterize antimicrobial resistance genes and mobile genetic elements associated with phenotypically positive ESBL $E$. coli isolates recovered from cattle and retail meat samples collected through the NARMS program between 2011 and 2015. This information will help to characterize the molecular epidemiology of CTX-M carrying E. coli isolates in food animals and animal products monitored by the NARMS program.

\section{Materials and Methods}

\section{Bacterial strains}

Eighteen phenotypically positive ESBL E. coli isolates recovered from cattle fecal samples $(n=5)$ and retail meats (chicken breast $[n=5]$, ground turkey $[n=6]$, ground beef $[n=1]$, and pork chops $[n=1])$ by the NARMS program between 2011 and 2015 were identified and selected for characterization. The isolates were identified from a total of $8,721 \mathrm{E}$. coli isolates recovered from fecal samples of healthy cattle and retail meat samples. The fecal isolates $(n=3,079)$ were recovered from healthy cattle as part of a NARMS on-farm pilot program to monitor antimicrobial resistance in foodborne pathogens. The retail meat $E$. coli isolates $(n=5,642)$ were recovered from chicken breast, chicken wing, pork chops, ground beef, and ground turkey.

\section{In vitro antimicrobial susceptibility testing}

The bacterial isolates were tested for antimicrobial drug susceptibility using the Sensititre ${ }^{\mathrm{TM}}$ semiautomated antimicrobial susceptibility system (ThermoFisher Scientific, Trek Diagnositics, Cleveland, $\mathrm{OH}$ ) following the manufacturer's instructions. The antimicrobials tested were as follows: amoxicillin/clavulanic acid (AUG), ampicillin (AMP), azithromycin (AZM), cefoxitin (FOX), ceftiofur (TIO), ceftriaxone (CRO), chloramphenicol (CHL), ciprofloxacin (CIP), gentamicin (GEN), nalidixic acid (NAL), streptomycin (STR), sulfamethoxazole (SMX), tetracycline (TET), and trimethoprim/sulfamethoxazole (SXT).

A total $321 \mathrm{E}$. coli isolates with minimum inhibitory concentrations (MICs) $\geq 8 \mu \mathrm{g} / \mathrm{mL}$ for ceftiofur and/or $\geq 4 \mu \mathrm{g}$ / $\mathrm{mL}$ for ceftriaxone for isolates recovered before 2015 and $\geq 2 \mu \mathrm{g} / \mathrm{mL}$ for isolates recovered in 2015 were selected for further testing with a second panel of $9 \beta$-lactam antimicrobials: aztreonam (ATM), cefquinome (CQN), imipenem (IMI), cefepime (FEP), piperacillin-tazobactam (TZP), ceftazidime (TAZ), ceftazidime-clavulanic acid (CAZ/ CLA), cefotaxime (FOT), and cefotaxime-clavulanic acid (CTX/CLA). The Clinical and Laboratory Standards Institute (CLSI) confirmatory test for ESBL production was used and is based on cefotaxime and ceftazidime MICs with and without clavulanic acid. Isolates showing a three or more twofold concentration decrease in the cefotaxime and ceftazidime MICs when tested in combination with clavulanate versus the MICs of cefotaxime and ceftazidime when tested alone are considered $\mathrm{ESBL}^{+20}$

E. coli ATCC 25922, Enterococcus faecalis ATCC 29212, Staphylococcus aureus ATCC 29213, Pseudomonas aeruginosa ATCC 27853, and Klebsiella pneumoniae ATCC 7000603 were used as quality control organisms for MIC determinations. Results were interpreted according to CLSI guidelines for broth microdilution methods with the exception of STR (NARMS resistance breakpoint, $\geq 32 \mu \mathrm{g} /$ $\mathrm{mL}$ ), AZM (NARMS resistance breakpoint, $\geq 32 \mu \mathrm{g} / \mathrm{mL}$ ), and CQN (NARMS resistance breakpoint, $\geq 32 \mu \mathrm{g} / \mathrm{mL}$ ). ${ }^{20}$

\section{Conjugation}

Conjugation experiments using a plate mating protocol were used to determine the transferability of resistance phenotypes and localize CTX-M genes to conjugative plasmids. We selected seven $E$. coli isolates that carried different CTX$\mathrm{M}$ genes [N36254PS (bla $\left.a_{\mathrm{CTX}-\mathrm{M}-32}\right)$, N36410PS (bla $\left.{ }_{\mathrm{CTX}-\mathrm{M}-27}\right)$, N37058PS (bla CTX-M-32), N40513 (bla $_{\text {CTX-M-1 }}$, N40607 (bla $\left.a_{\text {CTX-M-1 }}\right)$, N46045 (bla CTX-M-15 $_{15}$, and N51980 $\left(\right.$ bla $\left._{\text {CTX-M-14 }}\right)$ ] as donor cells. MAX Efficiency ${ }^{\circledR} \mathrm{DH} 5 \alpha^{\mathrm{TM}}$ E. coli Competent Cells (Invitrogen, Carlsbad, CA) were used as recipients. The donors and recipients were grown in $2 \mathrm{~mL}$ LB medium (Becton Dickinson, Sparks, MD) at $37^{\circ} \mathrm{C}$ in a shaker incubator for 16-18 hours. Ten microliters of donor cells were spotted on top of $10 \mu \mathrm{L}$ of recipient strain $(\mathrm{DH} 5 \alpha)$ on blood agar plates and incubated at $37^{\circ} \mathrm{C}$ overnight. Each co-culture was then scraped from the plate and resuspended in $1 \mathrm{~mL} \mathrm{LB}$ broth. Ceftiofur and nalidixic acid (Sigma-Aldrich, St. Louis, MO) were used as selective agents for the donor and recipient strains, respectively. Transconjugants were selected on LB agar containing nalidixic acid $(30 \mu \mathrm{g} / \mathrm{mL})$ and ceftiofur $(4 \mu \mathrm{g} /$ $\mathrm{mL}$ ). The MICs of donors, recipients, and transconjugants were determined using the Sensititre semiautomated antimicrobial susceptibility system. The $\beta$-lactam susceptibility testing panel was used to confirm the phenotype.

Five transconjugants from each experiment were tested for the presence of CTX-M genes using PCR primers designed for CTX-M-1 group (forward primer, 5'-ATGGTTA AAAAATCACTGCGTCAGT-3'; reverse primer, 5'-TTAC AAACCGTTGGTGAGATTTTAGCC-3') and CTX-M-9 group (forward primer, 5'-ATGGTGACAAAGAGAGTGC 
AACGG-3'; reverse primer, 5'-TTACAGCCCTTCGGCGA TGATTCT-3'). The expected amplicon fragment size for CTX-M group 1 and 9 was 876 and 846 bp, respectively. The PCR amplification conditions included initial denaturation at $95^{\circ} \mathrm{C}$ for 10 minutes, 30 cycles of denaturing at $94^{\circ} \mathrm{C}$ for 30 seconds, annealing at $58^{\circ} \mathrm{C}$ for 60 seconds, and extension at $72^{\circ} \mathrm{C}$ for 60 seconds, and followed by final extension at $72^{\circ} \mathrm{C}$ for 7 minutes.

\section{PCR-based plasmid replicon typing of transconjugants}

Genomic DNA was extracted using the DNeasy Blood and Tissue kit (Qiagen, Valencia, CA) following the manufacturer's instructions. Amplification of plasmid replicon targets was carried out following the protocol described by Johnson et al. ${ }^{21}$ with minor modifications for IncP characterization. For IncP, a simplex PCR with an annealing temperature of $65^{\circ} \mathrm{C}$ was used. The amplified products were separated by gel electrophoresis on $1.0 \%$ agarose gels.

\section{Whole-genome sequencing}

Whole-genome sequencing was used to characterize the resistome and plasmids in all strains $(n=18)$. Briefly, DNA was extracted using the DNeasy Blood and Tissue Kit (Qiagen) following the manufacturer's instructions. Wholegenome sequencing was performed on the MiSeq Desktop Sequencer using v2 sequencing reagent kits (Illumina, San Diego, CA). A de novo assembly was performed using CLC Genomics Workbench version 8.0 (Qiagen). Contigs of less than $200 \mathrm{bp}$ were removed from analysis. The number of assembled contigs ranged between 78 and 258 with an average coverage of $50 \times$.

\section{Resistome analysis}

Resistance genes were identified using BLASTX ${ }^{\mathrm{a}}$ and the ResFinder resistance gene database. ${ }^{22}$ The BLASTX results were processed with in-house PERL scripts to identify antimicrobial resistance genes using an $85 \%$ amino acid identity and $50 \%$ minimum sequence length.

\section{Phylogenetic analysis}

The Center for Food Safety and Applied Nutrition (CFSAN) SNP pipeline ${ }^{b}$ was used to create the single nucleotide polymorphism (SNP) matrices from sequence data for the phylogenetic analysis. SNP redundancy by linkage disequilibrium was reduced and the phylogenetic tree was constructed with the maximum likelihood algorithm using the SNPhylo package. $^{23}$

\section{Plasmid profiling}

Identification of the plasmid type was done using the PlasmidFinder database. ${ }^{\mathrm{c}}$ The cutoff threshold for identity was set at $95 \%$ to determine the existence for a particular plasmid.

\footnotetext{
${ }^{a}$ https://blast.ncbi.nlm.nih.gov/

bhttp://snp-pipeline.readthedocs.io/

${ }^{\mathrm{c}} \mathrm{https} / / /$ cge.cbs.dtu.dk/services/PlasmidFinder
}

\section{Multilocus sequence typing profiling}

E. coli multilocus sequence typing (MLST) allelic profiles and sequences were downloaded from the PubMLST database. ${ }^{\mathrm{d}}$ A total of 7,113 profiles for 7 different loci were used for the MLST. The SRST2 pipeline ${ }^{24}$ was used to determine the MLST type for our E. coli isolates.

\section{Nucleotide sequence accession numbers}

The whole-genome sequence data reported in this study have been deposited at DDBJ/ENA/GenBank under the following accession numbers: AZCE00000000, AZCG0000 0000, AZCB00000000, AZCC00000000, AZCD00000000, AZCF00000000, AZCH00000000, NTMS00000000, NT MT00000000, NTMU00000000, NTMV00000000, NTMW 00000000, NTMX00000000, NTMY00000000, NTMZ000000 00, NTNA00000000, NTNB00000000, and NTNC00000000.

\section{Results}

In vitro antimicrobial susceptibility testing of $\mathrm{E}$. coli isolates

The antimicrobial resistance profiles of phenotypically positive ESBL E. coli isolates are shown in Table 1. All the isolates were resistant to ampicillin, ceftiofur, ceftriaxone, and cefotaxime, as expected. Three of the 5 cattle and 2 of the 13 retail meat $E$. coli isolates were resistant to cefquinome, a fourth-generation cephalosporin. In addition, one of the cattle $E$. coli isolates and two of retail meat isolates showed resistance to aztreonam, a monobactam. Among the 18 strains producing ESBL, all had a three or more twofold concentration decrease in MIC for cefotaxime and ceftazidime in combination with clavulanic acid than the MIC when tested alone (Table 2). Other non- $\beta$-lactam resistances observed were to sulfisoxazole $(15 / 18)$, tetracycline $(14 / 18)$, chloramphenicol (3/18) streptomycin $(9 / 18)$, nalidixic acid (1/18), and trimethoprim/sulfamethoxazole (4/18).

\section{Conjugation and plasmid typing}

At least two plasmid types were detected using PlasmidFinder from each of the bla $_{\mathrm{CTX}-\mathrm{M}}{ }^{+} E$. coli isolates (Fig. 1). The conjugation results showed that bla $a_{\mathrm{CTX}-\mathrm{M}}$ genes can be transferred by broth mating. The transfer of bla $a_{\text {CTX-M }}$ gene was confirmed by PCR. We further confirmed the plasmid replicon type of the transconjugant with the ESBL phenotype using PCR-based replicon typing. Based on the replicon typing, the two conjugative plasmid types that carried the $C T X-M$ genes were IncI1 and IncF, present in isolates from chicken breast and cattle feces, respectively. One of the $b l a_{\mathrm{CTX}-\mathrm{M}}$ genes identified from cattle isolates (bla $a_{\mathrm{CTX}-\mathrm{M}-32}$ ) was not transferable by conjugation. Other resistance phenotypes co-transferred by conjugation include $\mathrm{Tet}^{\mathrm{R}}$ and $\mathrm{Smx}^{\mathrm{R}}$ (Table 1).

\section{Resistome analysis in ESBL E. coli isolates}

Characterization of resistance genes was conducted using whole-genome sequencing. The distribution of resistance genes is shown in Fig. 2. The ESBL genotypes in our

\footnotetext{
${ }^{\mathrm{d}}$ https://pubmlst.org/
} 
Table 1. Minimum Inhibitory Concentration Values of Extended-Spectrum Beta-Lactamase-Positive Escherichia coli Isolates Obtained from Fecal Samples of Healthy Cattle and Retail Meat Samples

\begin{tabular}{|c|c|c|c|c|c|c|c|c|c|c|c|c|c|c|c|}
\hline \multirow[b]{2}{*}{$I D$} & \multirow[b]{2}{*}{ Source } & \multicolumn{14}{|c|}{ Antimicrobials $^{\mathrm{a}}$} \\
\hline & & $\begin{array}{c}A M C \\
\geq 32 / 16\end{array}$ & $\begin{array}{c}A M P \\
\geq 32\end{array}$ & $\begin{array}{c}A Z M^{\mathrm{b}} \\
\geq 32\end{array}$ & $\begin{array}{l}F O X \\
\geq 32\end{array}$ & $\begin{array}{c}C R O \\
\geq 4\end{array}$ & $\begin{array}{c}M E M \\
\geq 4\end{array}$ & $\begin{array}{l}C H L \\
\geq 32\end{array}$ & $\begin{array}{l}C I P \\
\geq 4\end{array}$ & $\begin{array}{l}N A L \\
\geq 32\end{array}$ & $\begin{array}{l}G E N \\
\geq 16\end{array}$ & $\begin{array}{c}S T R^{\mathrm{b}} \\
\geq 32\end{array}$ & $\begin{array}{l}\text { TET } \\
\geq 16\end{array}$ & $\begin{array}{l}F I S \\
\geq 16\end{array}$ & $\begin{array}{c}S X T \\
\geq 4 / 76\end{array}$ \\
\hline N36254PS & Farm, fecal & 4 & $>32$ & 4 & 8 & 8 & $\leq 0.06$ & 8 & $\leq 0.015$ & 2 & 0.5 & 32 & $>32$ & $>256$ & $\leq 0.12$ \\
\hline N36410PS & Farm, fecal & 8 & $>32$ & 4 & 8 & $>64$ & $\leq 0.06$ & 8 & $\leq 0.015$ & 2 & 0.5 & 16 & $\leq 4$ & $>256$ & $>4$ \\
\hline N37058PS & Farm, fecal & 4 & $>32$ & 4 & 4 & 32 & $\leq 0.06$ & $>32$ & 1 & 4 & 0.5 & 32 & $>32$ & $>256$ & 0.25 \\
\hline N37122PS & Farm, fecal & 4 & $>32$ & 4 & 8 & $>64$ & $\leq 0.06$ & 8 & $\leq 0.015$ & 2 & 1 & 8 & $\leq 4$ & $>256$ & $>4$ \\
\hline N37139PS & Farm, fecal & 4 & $>32$ & 4 & 8 & 64 & $\leq 0.06$ & 8 & $\leq 0.015$ & 2 & 0.5 & 8 & $\leq 4$ & $>256$ & $>4$ \\
\hline N40513 & Chicken breast & 8 & $>32$ & 4 & 4 & 64 & $\leq 0.06$ & 4 & $\leq 0.015$ & 2 & 0.5 & 8 & $>32$ & $>256$ & 0.5 \\
\hline N40607 & Chicken breast & 8 & $>32$ & 2 & 8 & $>64$ & $\leq 0.06$ & 4 & $\leq 0.015$ & 4 & 0.5 & 16 & $>32$ & $>256$ & 0.5 \\
\hline N43684 & Chicken breast & 4 & $>32$ & 4 & 4 & $>64$ & $\leq 0.06$ & 4 & $\leq 0.015$ & 2 & 0.5 & 8 & $\leq 4$ & $>256$ & 1 \\
\hline N44807 & Ground turkey & 8 & $>32$ & 4 & 8 & $>64$ & $\leq 0.06$ & 8 & $\leq 0.015$ & 4 & $\leq 0.25$ & $>64$ & $>32$ & $\leq 16$ & $\leq 0.12$ \\
\hline N46045 & Ground beef & 32 & $>32$ & 4 & 8 & $>64$ & $\leq 0.06$ & $>32$ & $\leq 0.015$ & 4 & 0.5 & $>64$ & $>32$ & $>256$ & $>4$ \\
\hline N51980 & Pork chop & 8 & $>32$ & 4 & 4 & 64 & $\leq 0.06$ & 8 & $\leq 0.015$ & 2 & 0.5 & $>64$ & $>32$ & $\leq 16$ & $\leq 0.12$ \\
\hline N53976 & Ground turkey & 4 & $>32$ & 2 & 4 & 32 & 0.25 & 4 & 0.5 & 2 & 4 & 8 & $>32$ & $>256$ & 2 \\
\hline N56041 & Ground turkey & 16 & $>32$ & 2 & 4 & $>64$ & $\leq 0.06$ & $>32$ & 0.12 & $>32$ & 0.5 & $>64$ & 32 & $>256$ & $\leq 0.12$ \\
\hline N56738 & Ground turkey & 8 & $>32$ & 4 & 4 & $>64$ & $\leq 0.06$ & 8 & $\leq 0.015$ & 2 & 1 & 64 & $>32$ & $>256$ & $\leq 0.12$ \\
\hline N58201 & Chicken breast & 8 & $>32$ & 4 & 4 & 64 & $\leq 0.06$ & 4 & $\leq 0.015$ & 4 & 4 & 8 & $>32$ & $>256$ & 0.5 \\
\hline N60559 & Chicken breast & 8 & $>32$ & 4 & 8 & 64 & $\leq 0.06$ & 4 & $\leq 0.015$ & 2 & $>16$ & 4 & $>32$ & $>256$ & 1 \\
\hline N60592 & Ground turkey & 8 & $>32$ & 4 & 4 & 64 & $\leq 0.06$ & 4 & $\leq 0.015$ & 2 & 16 & 64 & $>32$ & $>256$ & $\leq 0.12$ \\
\hline N63148 & Ground turkey & 4 & $>32$ & 4 & 2 & 64 & $\leq 0.06$ & 8 & $\leq 0.015$ & 2 & 0.5 & 8 & 32 & $\leq 16$ & $\leq 0.12$ \\
\hline
\end{tabular}

${ }^{\mathrm{a}}$ CLSI breakpoint.

${ }^{\mathrm{b}} \mathrm{NARMS}$ breakpoint.

AMC, amoxicillin/clavulanic acid; AMP, ampicillin; AZM, azithromycin; CHL, chloramphenicol; CIP, ciprofloxacin; CRO, ceftriaxone; FIS, sulfisoxazole; FOX, cefoxitin; GEN, gentamicin; MEM, meropenem; NAL, nalidixic acid; STR, streptomycin; SXT, trimethoprim/ sulfamethoxazole; TET, tetracycline.

Table 2. Minimum Inhibitory Concentration Values from the Extended-Spectrum Beta-Lactamase Panel for Escherichia coli Isolates Obtained from Fecal Samples of Healthy Cattle and Retail Meat Samples with Diminished Susceptibility or Resistance to BroAd-Spectrum CEPHAlosporins

\begin{tabular}{|c|c|c|c|c|c|c|c|c|c|c|}
\hline & \multirow[b]{2}{*}{ Source } & \multicolumn{9}{|c|}{ Antimicrobials $^{\mathrm{a}}$} \\
\hline & & $\begin{array}{c}A T M \\
\geq 16\end{array}$ & $\begin{array}{l}F E P \\
\geq 16\end{array}$ & $\begin{array}{c}C T X \\
\geq 4\end{array}$ & $\begin{array}{c}C Q N^{\mathrm{b}} \\
\geq 32\end{array}$ & $\begin{array}{l}C A Z \\
\geq 16\end{array}$ & $\begin{array}{c}I P M \\
\geq 4\end{array}$ & $\begin{array}{c}T Z P \\
\geq 128 / 4\end{array}$ & $\begin{array}{c}C T X / C L A \\
-\end{array}$ & $\begin{array}{c}C A Z / C L A \\
-\end{array}$ \\
\hline N36254PS & Farm, fecal & 2 & 1 & 8 & 4 & 1 & 0.12 & $\leq 0.5$ & $\leq 0.06$ & $\leq 0.06$ \\
\hline N36410PS & Farm, fecal & 16 & 4 & 64 & $>32$ & 4 & 0.12 & 8 & 0.25 & 0.12 \\
\hline N37058PS & Farm, fecal & 8 & 4 & 32 & 16 & 4 & 0.25 & 1 & $\leq 0.06$ & 0.12 \\
\hline N37122PS & Farm, fecal & 8 & 8 & 128 & 32 & 2 & 0.12 & 1 & $\leq 0.06$ & 0.12 \\
\hline N37139PS & Farm, fecal & 8 & 4 & 64 & 32 & 8 & 0.12 & $\leq 0.5$ & $\leq 0.06$ & $\leq 0.06$ \\
\hline N40513 & Chicken breast & 8 & 4 & 16 & 8 & 1 & 0.25 & 1 & $\leq 0.06$ & 0.25 \\
\hline N40607 & Chicken breast & 16 & 8 & 64 & 16 & 8 & 0.25 & 2 & $\leq 0.06$ & 0.25 \\
\hline N43684 & Chicken breast & $>32$ & 16 & 128 & $>32$ & 8 & 0.25 & 2 & 0.12 & 0.25 \\
\hline N44807 & Ground turkey & 8 & 4 & 64 & 16 & 1 & 0.25 & 2 & 0.12 & 0.25 \\
\hline N46045 & Ground beef & 4 & 8 & 32 & 32 & 1 & 0.12 & 1 & $\leq 0.06$ & 0.25 \\
\hline N51980 & Pork chop & 4 & 2 & 32 & 8 & 4 & 0.12 & 2 & $\leq 0.06$ & 0.12 \\
\hline N53976 & Ground turkey & 8 & 8 & 32 & 16 & 1 & 0.25 & 2 & $\leq 0.06$ & 0.12 \\
\hline N56041 & Ground turkey & 4 & 4 & 16 & 16 & 2 & 0.25 & 2 & $\leq 0.06$ & 0.25 \\
\hline N56738 & Ground turkey & 4 & 4 & 32 & 16 & 4 & 0.12 & 2 & $\leq 0.06$ & 0.12 \\
\hline N58201 & Chicken breast & 4 & 2 & 16 & 8 & 2 & 0.12 & 2 & $\leq 0.06$ & $\leq 0.06$ \\
\hline N60559 & Chicken breast & 4 & 4 & 16 & 8 & 1 & 0.25 & 1 & $\leq 0.06$ & 0.25 \\
\hline N60592 & Ground turkey & 8 & 8 & 32 & 16 & 2 & 0.12 & 1 & $\leq 0.06$ & 0.25 \\
\hline N63148 & Ground turkey & 8 & 2 & 16 & 8 & 8 & 0.12 & 4 & 8 & 8 \\
\hline
\end{tabular}

${ }^{\mathrm{a} C L S I}$ breakpoint.

${ }^{\mathrm{b}}$ NARMS breakpoint.

ATM, aztreonam; CAZ, ceftazidime; CAZ/CLA, ceftazidime-clavulanic acid; CQN, cefquinome; CTX, cefotaxime; CTX/CLA, cefotaxime-clavulanic acid; FEP, cefepime; IPM, imipenem; TZP, piperacillin-tazobactam. 


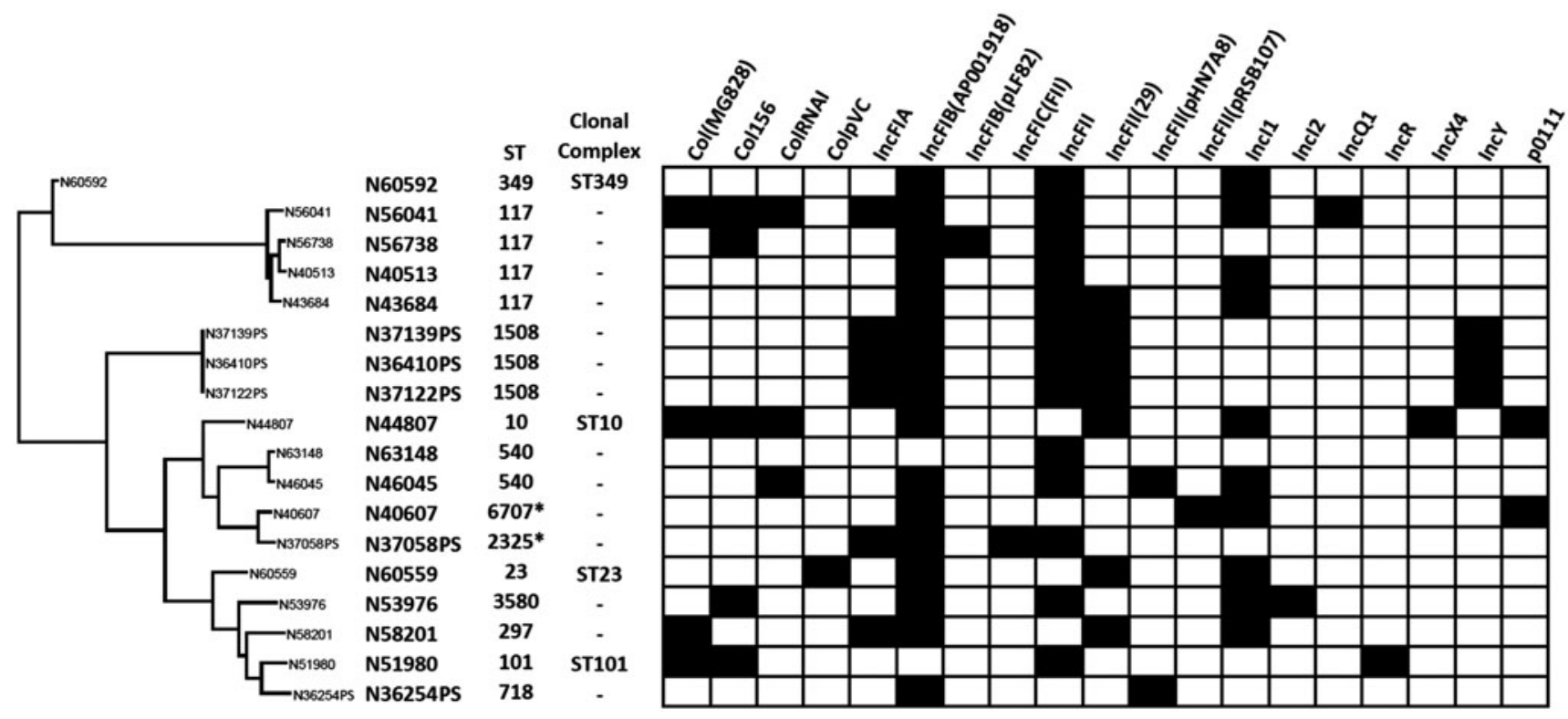

FIG. 1. Plasmid and MLST profiling of bla ${ }_{C T X-M}$-encoding Escherichia coli isolates. Phylogenetic tree generated based on the SNP analysis of the WGS data. The black color indicates the presence of the plasmid type based on plasmid finder. $6707^{*}$ - a single SNP on recA (T176G) and $2325^{*}$ — a single SNP on purA (G102A). MLST, multilocus sequence typing; SNP, single nucleotide polymorphism.

isolates were $b l a_{\mathrm{CTX}-\mathrm{M}-1}$, bla $a_{\mathrm{CTX}-\mathrm{M}-14}, b l a_{\mathrm{CTX}-\mathrm{M}-15,}$ bla $a_{\mathrm{CTX}-}$ M-27, and bla $a_{\mathrm{CTX}-\mathrm{M}-32}$. CTX-M $\beta$-lactamases exhibit increased hydrolytic activity against cefotaxime and ceftriaxone, but generally not against ceftazidime, which has important implications for laboratory detection. Similarly, in this study, all CTX-M-positive isolates were resistant to cefotaxime and none were resistant to ceftazidime. This is

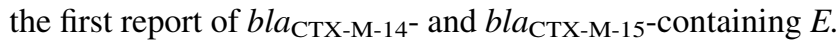

coli isolates from NARMS retail meat program in the United States.

The CTX-M genes identified in our isolates had 100\% nucleotide sequence identity with previously reported CTX-M genes. All five of the chicken breast $E$. coli isolates carried bla $_{\mathrm{CTX}-\mathrm{M}-1}$, four of the five cattle isolates (N36410PS, N37058PS, N37122PS, and N37139PS) had bla $a_{\text {CTX-M-27, }}$ and one cattle isolate (N36254PS) contained bla $a_{\mathrm{CTX}-\mathrm{M}-32}$.

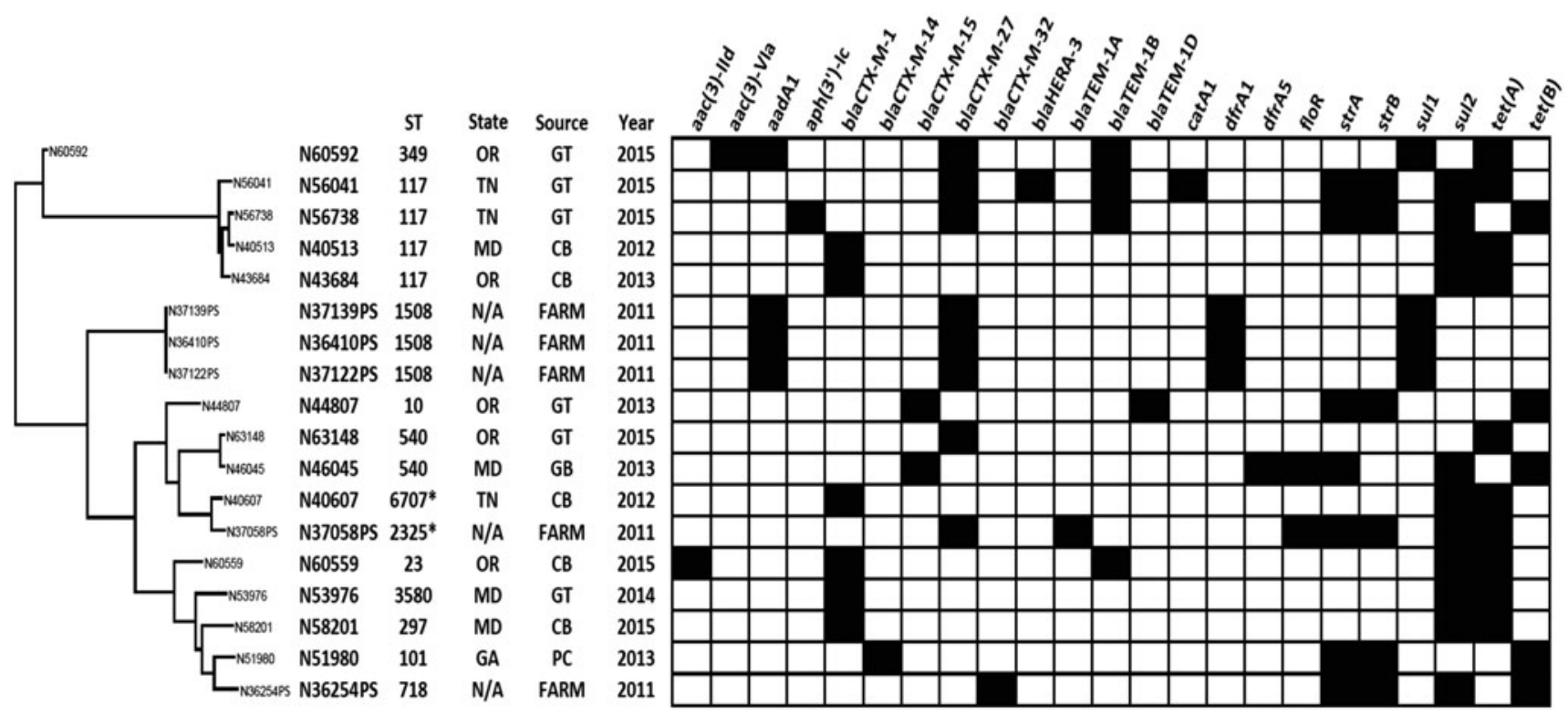

FIG. 2. Phylogenetic analysis and summary of MLST profiling and antimicrobial resistance genes identified in phenotypically positive ESBL E. coli isolates recovered from cattle and chicken breast. Clustering based on the SNP analysis of WGS data. The black color indicates the resistance gene identified. ESBL, extended-spectrum beta-lactamase.

${ }^{c}$ https://cge.cbs.dtu.dk/services/PlasmidFinder 

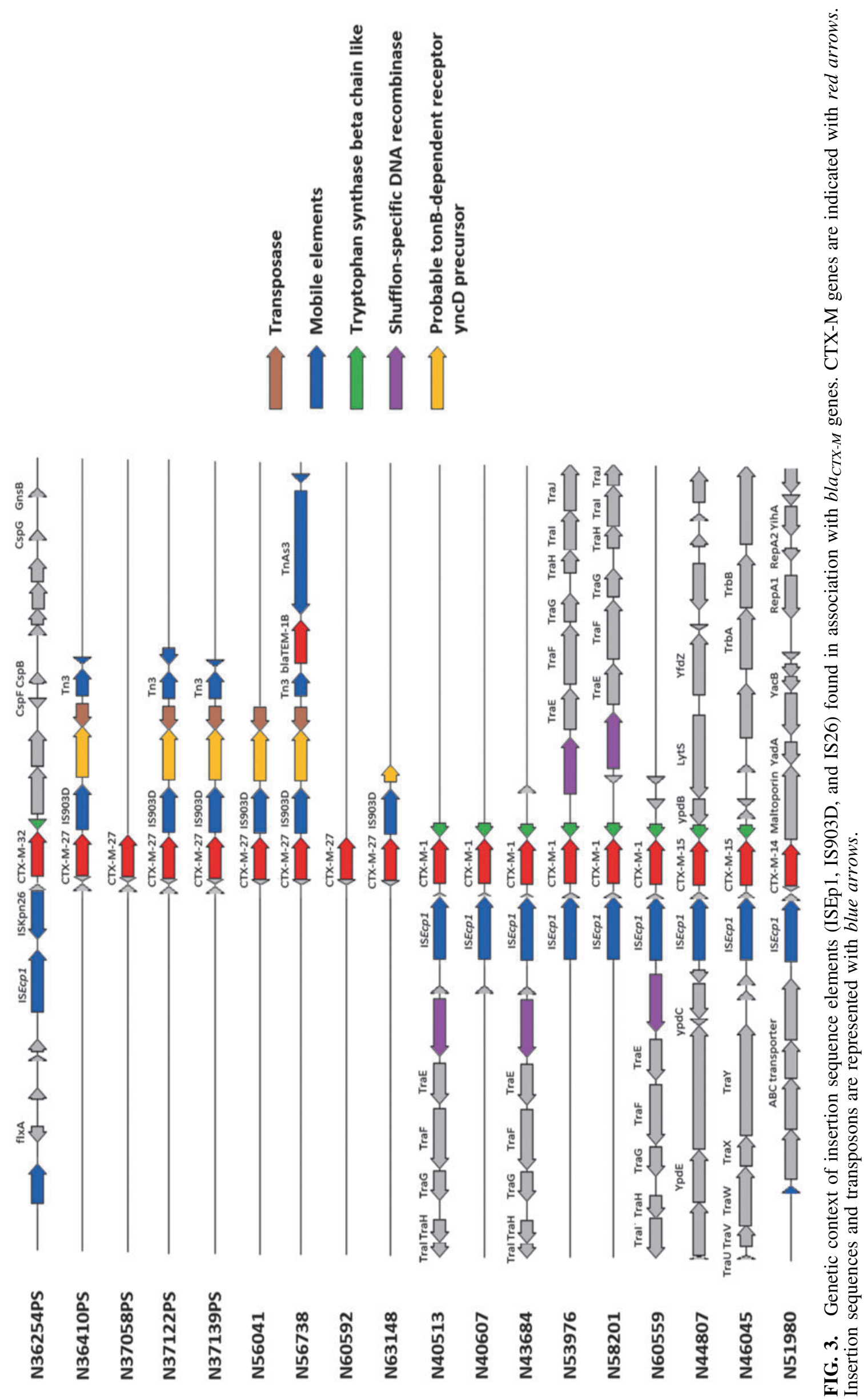
Three of the four $b l a_{\mathrm{CTX}-\mathrm{M}-27^{+}}$cattle $E$. coli isolates clustered together and had identical MLST type (ST1508) (Fig. 2).

$E$. coli isolates recovered from ground turkey carried diverse CTX-M genes, including bla $_{\mathrm{CTX}-\mathrm{M}-1}(n=1)$, bla $_{\mathrm{CTX}-\mathrm{M}-15}$ $(n=1)$, and $b l a_{\mathrm{CTX}-\mathrm{M}-27}(n=4)$. A single $E$. coli isolate each from pork chops and ground beef carried $b l a_{\mathrm{CTX}-\mathrm{M}-14}$ and $b l a_{\mathrm{CTX}-\mathrm{M}-15}$, respectively. The primary mechanisms responsible for the acquisition and mobilization of CTX-M genes are insertions sequences, transposons, and ISCRI. In our isolates, we identified ISEcp 1, IS26, and IS903-D mobilization elements (Fig. 3). In chicken breast isolates, bla $a_{\mathrm{CTX}-\mathrm{M}-1}$ gene was present as part of an ISEcpl transposition unit and shares sequence similarity with the backbone structure of the IncI plasmid. Conjugation results demonstrated that tet and sul resistance genes were carried on the same IncI plasmids harboring bla $a_{\mathrm{CTX}-\mathrm{M}-1}$ gene.

Streptomycin-resistant isolates ( MIC $\geq 32$ ) carried one or more aminoglycoside resistance genes. The aminoglycoside resistance genes commonly identified in these isolates were $\operatorname{str} A\left[\operatorname{aph}\left(3^{\prime}\right)-I b\right], \operatorname{str} B\left[\operatorname{aph}\left(6^{\prime}\right)-I d\right]$, and $\operatorname{aadA} 1$. One isolate with a gentamicin MIC $>16 \mu \mathrm{g} / \mathrm{mL}$ possessed an aac(3)-IId gene. Three E. coli isolates (N36410PS, N37122PS, and N37139PS) contained a class 1 integron carrying aadA1, $d f r A$, and qacE genes.

Two chloramphenicol-resistant isolates carried the floR gene and one contained catA1. All, but three E. coli isolates were resistant to sulfisoxazole and had either sull or sul2. Four also carried the dihydrofolate reductase gene $d f r A$.

\section{Discussion}

CTX-M-producing strains appear to have quickly spread worldwide, with the notable exception of the United States where TEM- and SHV-type ESBL have appeared to predominate until recently. CTX-M ESBLs have been reported in the United States mainly from human clinical isolates of Enterobacteriaceae encoding for CTX-M group 1 and 9. 7,10,16, 25,26

Infections caused by bacteria producing CTX-M enzymes are not limited to the hospital setting. ${ }^{27}$ Intestinal carriage of CTX-M-producing bacteria in food-producing animals and contamination of retail meat may contribute to increased incidences of infections with ESBL-producing bacteria in humans. Various reports have documented dissemination of ESBL-producing $E$. coli in healthy food-producing animals and animal products in several countries, ${ }^{12,13,17,28,29}$ and the potential of wild birds as possible reservoirs and vehicles for dissemination of CTX-Ms in the United States. ${ }^{15}$

In this study, we are reporting the first $b l a_{\mathrm{CTX}-\mathrm{M}-14}$ and $b_{\text {bTX-M-15 }}$ gene carrying $E$. coli isolate from NARMS retail meat program. All E. coli isolates obtained before 2011 from NARMS were phenotypically and genotypically negative for bla $_{\mathrm{CTX}-\mathrm{M} \cdot}{ }^{30}$ However, McDermott et al. recently identified the first bla $_{\mathrm{CTX}-\mathrm{M}-1 \text {-positive Salmonella isolate }}$ recovered from NARMS retail meat samples in the United States. ${ }^{18}$ Salmonella enterica serovar Infantis isolates containing bla $_{\mathrm{CTX}-\mathrm{M}-65}$ obtained from chicken, cattle, and human sources collected between 2012 and 2015 in the United States through routine NARMS surveillance have been re-

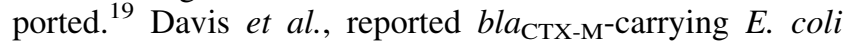
strains among isolates collected from Washington State cattle in 2011, while none from those collected in 2008 . $^{13}$ Investigations of nontyphoidal Salmonella isolates of human origin submitted to Center for Disease Control and Prevention (CDC) as part of the NARMS program between 2005 and 2007 identified Salmonella isolates producing CTX-M enzymes $\left(\text { bla }_{\mathrm{CTX}-\mathrm{M}-15}, \text { bla }_{\mathrm{CTX}-\mathrm{M}-5} \text {, and } \text { bla }_{\mathrm{CTX}-\mathrm{M}-55 / 57)}\right)^{31,32}$

The successful spread of CTX-M genes depends on the clonal nature of strains carrying the resistance genes, and mobile genetic elements responsible for its capture and spread. E. coli ST131 and ST405 are by far the most important sequence types (STs) associated with the sudden worldwide increase of CTX-M genes, including $b l a_{\mathrm{CTX}-\mathrm{M}-15}{ }^{33}$ and other CTX-M genes such as $b l a_{\mathrm{CTX}-\mathrm{M}-1}$, bla $a_{\mathrm{CTX}-\mathrm{M}-3}$, bla $_{\mathrm{CTX}-\mathrm{M}-10}$, and bla $_{\mathrm{CTX}-\mathrm{M}-14 \cdot{ }^{26}}$ None of our bla ${ }_{\text {CTX-M }}{ }^{+} E$. coli belonged to ST131 or ST405, indicating that the spread of CTX-M genes is not associated with the established clonal strains. We observed diverse STs carrying the same $b l a_{\mathrm{CTX}-\mathrm{M}}$ gene, and in some instances, different $b l a_{\mathrm{CTX}-\mathrm{M}}$ genes carried by the same ST. For example, E. coli isolates recovered from two ground turkey isolates carrying $b l a_{\mathrm{CTX}}$ M-27 and two chicken breast isolates carrying $b l a_{\mathrm{CTX}-\mathrm{M}-1}$ were ST117, indicating the potential of the same ST to spread multiple CTX-M genes.

The most commonly reported mobilization elements mediating the spread of CTX-M genes include ISEcp 1, ISCR1, and IS $26^{34-36}$ and phage-related sequences. ${ }^{37}$ In our isolates, different IS elements, including ISEcp1, IS26, and IS903-D were identified upstream of $b l a_{\mathrm{CTX}-\mathrm{M}-1}, b l a_{\mathrm{CTX}-\mathrm{M}-27}$, and $b l a_{\mathrm{CTX}-\mathrm{M}-32}$ genes as previously reported elsewhere. ${ }^{38-40}$ All phenotypically positive ESBL $E$. coli isolates recovered from chicken breast encoded $b l a_{\mathrm{CTX}-\mathrm{M}-1}$ as part of an ISEcpl transposition unit and shares sequence similarity with the backbone structure of the IncI plasmid. IncI1 has been shown to be one of the main plasmid lineages that contribute to the dissemination of $b l a_{\mathrm{CTX}-\mathrm{M}-1}$ genes in the food chain, including chicken retail meat, the environment, and humans. ${ }^{41}$ In the Netherlands, bacteria producing ESBL isolated from chicken meat and gut of broilers predominantly carried bla $_{\mathrm{CTX}-\mathrm{M}-1}$ located on IncI1 plasmids. ${ }^{42}$ Similarly, Day et al. demonstrated a widespread distribution of IncI1 plasmids carrying bla $_{\text {CTX-M-1 }}$ gene among $E$. coli recovered from humans, animals, and food products in Germany, the Netherlands, and the United Kingdom. ${ }^{43}$ Furthermore, a study in the Netherlands revealed the presence of indistinguishable genotypes, CTX-M genes and plasmids, in E. coli obtained from poultry, retail chicken meat, and human clinical samples, suggesting possible exchange through food chain. ${ }^{12}$

The $b l a_{\mathrm{CTX}-\mathrm{M}-27}$ genes identified in our cattle and ground turkey $E$. coli isolates were associated with $\mathrm{IncF}$ plasmids. Horizontal transfer is important in the dissemination of bla $a_{\mathrm{CTX}-\mathrm{M}-27}$ gene, as evidenced by the fact that $b l a_{\mathrm{CTX}-\mathrm{M}-27}$ genes in our isolates were transferred by conjugation, confirming the location of the gene on a conjugative plasmid. IncF plasmids encode numerous addiction systems that ensure and contribute to the maintenance of antimicrobial resistance determinants and virulence factors even in the absence of antibiotic selection pressure. ${ }^{44}$ IncF replicon-type plasmids carrying $b a_{\mathrm{CTX}-\mathrm{M}-27}$ have been documented in cefotaximaseproducing $E$. coli clinical isolates from Dublin, Ireland. ${ }^{45}$ Plasmids carrying $b l a_{\mathrm{CTX}-\mathrm{M}}$ genes are often self-conjugative and carry additional resistance determinants, ${ }^{46}$ greatly facilitating widespread distribution of alleles in different environments. A recent report has documented a case of ceftriaxone treatment failure caused by Salmonella Typhimurium due to 
the in vivo acquisition of a bla $a_{\mathrm{CTX}-\mathrm{M}-27-\mathrm{encoding} \text { IncFII group }}$ transmissible plasmid. ${ }^{47}$

The presence of $b l a_{\mathrm{CTX}-\mathrm{M}-15}$ and $b l a_{\mathrm{CTX}-\mathrm{M}-14}$ was reported in most U.S. medical centers participating in the Meropenem Yearly Susceptibility Test Information Collection (MYSTIC) program in 2007. ${ }^{10,25}$ In this study, we are reporting for the first time $b l a_{\mathrm{CTX}-\mathrm{M}-15^{-}}$and $b l a_{\mathrm{CTX}-\mathrm{M}-14^{-}}$ encoding E. coli from retail meat samples collected in 2013. CTX-M-15 and CTX-M-14, the two most frequently identified CTX-M enzyme worldwide, have been detected in bacteria isolated from humans, animals, and the environment. ${ }^{17,42,48}$ A recent study from six community hospitals in North Carolina and Virginia from 2010 to 2012 demonstrated that $80 \%$ of ESBL-producing isolates contained CTX-M enzymes. In these isolates, ST131 was associated with $48 \%$ of $b l a_{\mathrm{CTX}-\mathrm{M}-15}$-producing $E$. coli isolates and $66 \%$

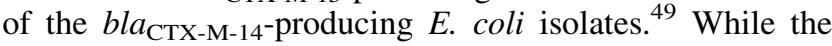
prevalence of these two successful CTX-M enzymes is low from domestic food animal sources, monitoring will continue to help determine whether this mechanism is becoming more widespread among animal and food strains of $E$. coli in the United States.

\section{Acknowledgments}

We thank NARMS participating state and local health departments, USDA-ARS, and University partners involved in sample collection. We would also like to thank Dr. Maureen Davidson for critical review of the article before submission. This work was supported by the U.S. Food and Drug Administration with internal funds as part of routine work. The views expressed in this article are those of the authors and do not necessarily reflect the official policy of the Department of Health and Human Services, the U.S. Food and Drug Administration, the U.S. Department of Agriculture, or the U.S. Government. Mention of trade names or commercial products in this publication is solely for the purpose of providing specific information and does not imply recommendation or endorsement by the U.S. Food and Drug Administration or the USDA. The USDA is an equal opportunity provider and employer.

\section{Disclosure Statement}

G.H.L. has accepted consulting fees for service on scientific advisory boards, honoraria, and travel expenses from various manufacturers of animal pharmaceuticals.

\section{References}

1. Bradford, P.A. 2001. Extended-spectrum beta-lactamases in the 21st century: characterization, epidemiology, and detection of this important resistance threat. Clin. Microbiol. Rev. 14:933-951.

2. Paterson, D.L., and R.A. Bonomo. 2005. Extended-spectrum beta-lactamases: a clinical update. Clin. Microbiol. Rev. 18: 657-686.

3. Shah, P.M., and W. Stille. 1983. Escherichia coli and Klebsiella pneumoniae strains more susceptible to cefoxitin than to third generation cephalosporins. J. Antimicrob. Chemother. 11:597-598.

4. CDC. 2013. Antibiotic Resistance Threats in the United States, 2013. Centers for Disease Control and Prevention.
Atlanta, GA. Available at www.cdc.gov/drugresistance/ pdf/ar-threats-2013-508.pdf

5. Woodford, N., A. Carattoli, E. Karisik, A. Underwood, M.J. Ellington, and D.M. Livermore. 2009. Complete nucleotide sequences of plasmids pEK204, pEK499, and pEK516, encoding CTX-M enzymes in three major Escherichia coli lineages from the United Kingdom, all belonging to the international O25:H4-ST131 clone. Antimicrob. Agents Chemother. 53:4472-4482.

6. Pitout, J.D., P. Nordmann, K.B. Laupland, and L. Poirel. 2005. Emergence of Enterobacteriaceae producing extendedspectrum beta-lactamases (ESBLs) in the community. J. Antimicrob. Chemother. 56:52-59.

7. Lewis, J.S., M. Herrera, B. Wickes, J.E. Patterson, and J.H. Jorgensen. 2007. First report of the emergence of CTX-Mtype extended-spectrum beta-lactamases (ESBLs) as the predominant ESBL isolated in a U.S. health care system. Antimicrob. Agents Chemother. 51:4015-4021.

8. Canton, R., A. Novais, A. Valverde, E. Machado, L. Peixe, F. Baquero, and T.M. Coque. 2008. Prevalence and spread of extended-spectrum beta-lactamase-producing Enterobacteriaceae in Europe. Clin. Microbiol. Infect. 14(Suppl 1): 144-153.

9. D'Andrea, M.M., F. Arena, L. Pallecchi, and G.M. Rossolini. 2013. CTX-M-type beta-lactamases: a successful story of antibiotic resistance. Int. J. Med. Microbiol. 303: 305-317.

10. Castanheira, M., R.E. Mendes, P.R. Rhomberg, and R.N. Jones. 2008. Rapid emergence of blaCTX-M among Enterobacteriaceae in U.S. Medical Centers: molecular evaluation from the MYSTIC Program (2007). Microb. Drug Resist. 14:211-216.

11. Wittum, T.E., D.F. Mollenkopf, J.B. Daniels, A.E. Parkinson, J.L. Mathews, P.R. Fry, M.J. Abley, and W.A. Gebreyes. 2010. CTX-M-type extended-spectrum beta-lactamases present in Escherichia coli from the feces of cattle in Ohio, United States. Foodborne Pathog. Dis. 7:1575-1579.

12. Leverstein-van Hall, M.A., C.M. Dierikx, S.J. Cohen, G.M. Voets, M.P. van den Munckhof, A. van Essen-Zandbergen, T. Platteel, A.C. Fluit, N. Sande-Bruinsma, J. Scharinga, M.J. Bonten, D.J. Mevius; National ESBL Surveillance Group. 2011. Dutch patients, retail chicken meat and poultry share the same ESBL genes, plasmids and strains. Clin. Microbiol. Infect. 17:873-880.

13. Davis, M.A., W.M. Sischo, L.P. Jones, D.A. Moore, S. Ahmed, D.M. Short, and T.E. Besser. 2015. Recent emergence of Escherichia coli with cephalosporin resistance conferred by blaCTX-M on Washington State dairy farms. Appl. Environ. Microbiol. 81:4403-4410.

14. Carattoli, A., S. Lovari, A. Franco, G. Cordaro, P. Di Matteo, and A. Battisti. 2005. Extended-spectrum betalactamases in Escherichia coli isolated from dogs and cats in Rome, Italy, from 2001 to 2003. Antimicrob. Agents Chemother. 49:833-835.

15. Poirel, L., A. Potron, C. De La Cuesta, T. Cleary, P. Nordmann, and L.S. Munoz-Price. 2012. Wild coastline birds as reservoirs of broad-spectrum-beta-lactamaseproducing Enterobacteriaceae in Miami Beach, Florida. Antimicrob. Agents Chemother. 56:2756-2758.

16. Moland, E.S., J.A. Black, A. Hossain, N.D. Hanson, K.S. Thomson, and S. Pottumarthy. 2003. Discovery of CTX-Mlike extended-spectrum beta-lactamases in Escherichia coli isolates from five US States. Antimicrob. Agents Chemother. 47:2382-2383. 
17. Cottell, J.L., M.A. Webber, N.G. Coldham, D.L. Taylor, A.M. Cerdeno-Tarraga, H. Hauser, N.R. Thomson, M.J. Woodward, and L.J. Piddock. 2011. Complete sequence and molecular epidemiology of IncK epidemic plasmid encoding blaCTX-M-14. Emerg. Infect. Dis. 17:645-652.

18. McDermott, P.F., G.H. Tyson, C. Kabera, Y. Chen, C. Li, J.P. Folster, S.L. Ayers, C. Lam, H.P. Tate, and S. Zhao. 2016. Whole-genome sequencing for detecting antimicrobial resistance in nontyphoidal Salmonella. Antimicrob. Agents Chemother. 60:5515-5520.

19. Tate, H., J.P. Folster, C.H. Hsu, J. Chen, M. Hoffmann, C. Li, C. Morales, G.H. Tyson, S. Mukherjee, A.C. Brown, A. Green, W. Wilson, U. Dessai, J. Abbott, L. Joseph, J. Haro, S. Ayers, P.F. McDermott, and S. Zhao. 2017. Comparative analysis of extended-spectrum-beta-lactamase CTX-M-65producing Salmonella enterica serovar Infantis isolates from humans, food animals, and retail chickens in the United States. Antimicrob. Agents Chemother. 61:e00488-17.

20. CLSI. 2017. Performance Standards for Antimicrobial Susceptibility Testing; 27th Informational Supplement. CLSI Document M100-S27. Clinical and Laboratory Standards Institute, Wayne, PA.

21. Johnson, T.J., Y.M. Wannemuehler, S.J. Johnson, C.M. Logue, D.G. White, C. Doetkott, and L.K. Nolan. 2007. Plasmid replicon typing of commensal and pathogenic Escherichia coli isolates. Appl. Environ. Microbiol. 73:1976-1983.

22. Zankari, E., H. Hasman, S. Cosentino, M. Vestergaard, S. Rasmussen, O. Lund, F.M. Aarestrup, and M.V. Larsen. 2012. Identification of acquired antimicrobial resistance genes. J. Antimicrob. Chemother. 67:2640-2644.

23. Lee, T.H., H. Guo, X. Wang, C. Kim, and A.H. Paterson. 2014. SNPhylo: a pipeline to construct a phylogenetic tree from huge SNP data. BMC Genomics 15:162.

24. Inouye, M., H. Dashnow, L.A. Raven, M.B. Schultz, B.J. Pope, T. Tomita, J. Zobel, and K.E. Holt. 2014. SRST2: rapid genomic surveillance for public health and hospital microbiology labs. Genome Med. 6:90.

25. Castanheira, M., H.S. Sader, and R.N. Jones. 2010. Antimicrobial susceptibility patterns of KPC-producing or CTX-M-producing Enterobacteriaceae. Microb. Drug Resist. 16:61-65.

26. Naseer, U., and A. Sundsfjord. 2011. The CTX-M conundrum: dissemination of plasmids and Escherichia coli clones. Microb. Drug Resist. 17:83-97.

27. Pitout, J.D., and K.B. Laupland. 2008. Extended-spectrum beta-lactamase-producing Enterobacteriaceae: an emerging public-health concern. Lancet Infect. Dis. 8:159-166.

28. Horton, R.A., L.P. Randall, E.L. Snary, H. Cockrem, S. Lotz, H. Wearing, D. Duncan, A. Rabie, I. McLaren, E. Watson, R.M. La Ragione, and N.G. Coldham. 2011. Fecal carriage and shedding density of CTX-M extendedspectrum \{beta\}-lactamase-producing Escherichia coli in cattle, chickens, and pigs: implications for environmental contamination and food production. Appl. Environ. Microbiol. 77:3715-3719.

29. Tamang, M.D., H.M. Nam, T.S. Kim, G.C. Jang, S.C. Jung, and S.K. Lim. 2011. Emergence of extended-spectrum beta-lactamase (CTX-M-15 and CTX-M-14)-producing nontyphoid Salmonella with reduced susceptibility to ciprofloxacin among food animals and humans in Korea. J. Clin. Microbiol. 49:2671-2675.

30. Zhao, S., K. Blickenstaff, A. Glenn, S.L. Ayers, S.L. Friedman, J.W. Abbott, and P.F. McDermott. 2009. Betalactam resistance in Salmonella strains isolated from retail meats in the United States by the National Antimicrobial Resistance Monitoring System between 2002 and 2006. Appl. Environ. Microbiol. 75:7624-7630.

31. Sjolund-Karlsson, M., R. Howie, A. Krueger, R. Rickert, G. Pecic, K. Lupoli, J.P. Folster, and J.M. Whichard. 2011. CTX-M-producing non-Typhi Salmonella spp. isolated from humans, United States. Emerg. Infect. Dis. 17:97-99.

32. Sjolund-Karlsson, M., R. Rickert, C. Matar, G. Pecic, R.L. Howie, K. Joyce, F. Medalla, E.J. Barzilay, and J.M. Whichard. 2010. Salmonella isolates with decreased susceptibility to extended-spectrum cephalosporins in the United States. Foodborne Pathog. Dis. 7:1503-1509.

33. Peirano, G., and J.D. Pitout. 2010. Molecular epidemiology of Escherichia coli producing CTX-M beta-lactamases: the worldwide emergence of clone ST131 O25:H4. Int. J. Antimicrob. Agents 35:316-321.

34. Toleman, M.A., P.M. Bennett, and T.R. Walsh. 2006. ISCR elements: novel gene-capturing systems of the 21st century? Microbiol. Mol. Biol. Rev. 70:296-316.

35. Cullik, A., Y. Pfeifer, R. Prager, H. von Baum, and W. Witte. 2010. A novel IS26 structure surrounds blaCTX-M genes in different plasmids from German clinical Escherichia coli isolates. J. Med. Microbiol. 59:580-587.

36. Partridge, S.R., J.A. Ellem, S.G. Tetu, Z. Zong, I.T. Paulsen, and J.R. Iredell. 2011. Complete sequence of pJIE143, a pir-type plasmid carrying ISEcp1-blaCTX-M-15 from an Escherichia coli ST131 isolate. Antimicrob. Agents Chemother. 55:5933-5935.

37. Oliver, A., T.M. Coque, D. Alonso, A. Valverde, F. Baquero, and R. Canton. 2005. CTX-M-10 linked to a phage-related element is widely disseminated among Enterobacteriaceae in a Spanish hospital. Antimicrob. Agents Chemother. 49:15671571.

38. Bou, G., M. Cartelle, M. Tomas, D. Canle, F. Molina, R. Moure, J.M. Eiros, and A. Guerrero. 2002. Identification and broad dissemination of the CTX-M-14 beta-lactamase in different Escherichia coli strains in the northwest area of Spain. J. Clin. Microbiol. 40:4030-4036.

39. Chanawong, A., F.H. M'Zali, J. Heritage, J.H. Xiong, and P.M. Hawkey. 2002. Three cefotaximases, CTX-M-9, CTX-M-13, and CTX-M-14, among Enterobacteriaceae in the People's Republic of China. Antimicrob. Agents Chemother. 46:630-637.

40. Dutour, C., R. Bonnet, H. Marchandin, M. Boyer, C. Chanal, D. Sirot, and J. Sirot. 2002. CTX-M-1, CTX-M-3, and CTXM-14 beta-lactamases from Enterobacteriaceae isolated in France. Antimicrob. Agents Chemother. 46:534-537.

41. Zurfluh, K., G. Jakobi, R. Stephan, H. Hachler, and M. Nuesch-Inderbinen. 2014. Replicon typing of plasmids carrying bla CTX-M-1 in Enterobacteriaceae of animal, environmental and human origin. Front. Microbiol. 5:555.

42. Fischer, E.A., C.M. Dierikx, A. van Essen-Zandbergen, H.J. van Roermund, D.J. Mevius, A. Stegeman, and D. Klinkenberg. 2014. The IncI1 plasmid carrying the blaCTX-M-1 gene persists in in vitro culture of a Escherichia coli strain from broilers. BMC Microbiol. 14:77.

43. Day, M.J., I. Rodriguez, A. van Essen-Zandbergen, C. Dierikx, K. Kadlec, A.K. Schink, G. Wu, M.A. Chattaway, V. DoNascimento, J. Wain, R. Helmuth, B. Guerra, S. Schwarz, J. Threlfall, M.J. Woodward, N. Coldham, D. Mevius, and N. Woodford. 2016. Diversity of STs, plasmids and ESBL genes among Escherichia coli from humans, animals and food in Germany, the Netherlands and the UK. J. Antimicrob. Chemother. 71:1178-1182. 
44. Carattoli, A. 2011. Plasmids in Gram negatives: molecular typing of resistance plasmids. Int. J. Med. Microbiol. 301: 654-658.

45. Burke, L., H. Humphreys, and D. Fitzgerald-Hughes. 2016. The molecular epidemiology of resistance in cefotaximaseproducing Escherichia coli clinical isolates from Dublin, Ireland. Microb. Drug Resist. 22:552-558.

46. Rossolini, G.M., M.M. D'Andrea, and C. Mugnaioli. 2008. The spread of CTX-M-type extended-spectrum betalactamases. Clin. Microbiol. Infect. 14(Suppl 1):33-41.

47. McCollister, B., C.V. Kotter, D.N. Frank, T. Washburn, and M.G. Jobling. 2016. Whole-genome sequencing identifies in vivo acquisition of a blaCTX-M-27-carrying IncFII transmissible plasmid as the cause of ceftriaxone treatment failure for an invasive Salmonella enterica serovar Typhimurium infection. Antimicrob. Agents Chemother. 60:7224-7235.

48. Hawkey, P.M., and A.M. Jones. 2009. The changing epidemiology of resistance. J. Antimicrob. Chemother. 64(Suppl 1): i3-i10.
49. Chen, L.F., J.T. Freeman, B. Nicholson, A. Keiger, S. Lancaster, M. Joyce, C.W. Woods, E. Cook, L. Adcock, S. Louis, A.L. Cromer, D.J. Sexton, and D.J. Anderson. 2014. Widespread dissemination of CTX-M-15 genotype extendedspectrum-beta-lactamase-producing Enterobacteriaceae among patients presenting to community hospitals in the southeastern United States. Antimicrob. Agents Chemother. 58:1200-1202.

Address correspondence to: Daniel A. Tadesse, DVM, PhD Division of Animal and Food Microbiology U.S. FDA, CVM 8401 Muirkirk Road Laurel, MD 20708

E-mail: daniel.tadesse@fda.hhs.gov 